\title{
Detection and high-resolution spectroscopy of a huge flare on the old M 9 dwarf DENIS 104814.7-395606.1^
}

\author{
B. Fuhrmeister and J. H. M. M. Schmitt
}

\author{
Hamburger Sternwarte, University of Hamburg, Gojenbergsweg 112, 21029 Hamburg, Germany \\ Received 7 November 2003 / Accepted 14 March 2004
}

\begin{abstract}
We report a flare on the M 9 dwarf DENIS 104814.7-395606.1, whose mass places it directly at the hydrogen burning limit. The event was observed in a spectral sequence during $1.3 \mathrm{~h}$. Line shifts to bluer wavelengths were detected in $\mathrm{H}_{\alpha}, \mathrm{H}_{\beta}$, and in the Na I D lines, indicating mass motions. In addition we detect a flux enhancement on the blue side of the two Balmer lines in the last spectrum of our series. We interpret this as rising gas cloud with a projected velocity of about $100 \mathrm{~km} \mathrm{~s}^{-1} \mathrm{which}$ may lead to mass ejection. The higher Balmer lines $\mathrm{H}_{\gamma}$ to $\mathrm{H}_{8}$ are not seen due to our instrumental setup, but in the last spectrum there is strong evidence for $\mathrm{H}_{9}$ being in emission.
\end{abstract}

Key words. stars: activity - stars: flare - stars: late-type

\section{Introduction}

DENIS 104814.7-395606.1 (hereafter DENIS 1048-39) was discovered in the DEep Near Infrared Survey (DENIS), which covered the southern sky (Epchtein 1997) in two near infrared bands $\left(J\right.$ and $K_{\mathrm{s}}$ ) and one optical band $(I)$. With this choice of bands DENIS is very sensitive to very low mass stars and brown dwarfs and thus excellently suited for searches for hitherto unknown low mass stellar or substellar objects in the solar vicinity. With a distance of only $4.6 \pm 0.3 \mathrm{pc}$ (Neuhäuser et al. 2002) DENIS 1048-39 is extremely close to the Sun. Classified as an M9 star, the lithium resonance line at $6708 \AA$ could not be detected, therefore substantial lithium depletion must have taken place (Delfosse et al. 2001). Compared to LP 944-20, which is also classified as M9, DENIS 1048-39 should be older and more massive. Theoretical models place DENIS 1048-39 directly at the hydrogen burning boundary with an estimated mass of 0.075 up to $0.09 M_{\text {Sun }}$ and an age of 1-2 Gyr (Neuhäuser et al. 2002) assuming a solar chemical composition. Thus DENIS 1048-39 may either be among the most massive brown dwarfs or among the least massive stars.

Interestingly, the $\mathrm{H}_{\alpha}$-line was found to be variable (Delfosse et al. 2001), implying that DENIS 1048-39 exhibits activity despite its old age and low mass. There are only a few very late type objects showing strong and persistent $\mathrm{H}_{\alpha}$ emission. $\mathrm{H}_{\alpha}$ emission, a well established activity indicator, is not detected in the majority of these objects. Instead one observes a steep decline of the strength of $\mathrm{H}_{\alpha}$ emission for stars later

Send offprint requests to: B. Fuhrmeister, e-mail: bfuhrmeister@hs.uni-hamburg.de

* Based on observations collected at the European Southern Observatory, Paranal, 68.D-0166A, Chile. than spectral type M7. This effect shows up in smaller equivalent widths (EW) of the $\mathrm{H}_{\alpha}$ line for these objects. However, since the $\mathrm{H}_{\alpha}$ line is seen against an increasingly faint photosphere for later objects, a better activity indicator is the ratio of the $\mathrm{H}_{\alpha}$ luminosity to the bolometric luminosity. This ratio was found to drop in only three subclasses (M 8-L 1) by one order of magnitude (Gizis et al. 2000). The same authors also found that the activity of these late type objects is primarily related to temperature and shows only a secondary dependence on mass and age. Thus strong $\mathrm{H}_{\alpha}$ emission does not necessarily imply youth; on the contrary, strong $\mathrm{H}_{\alpha}$ emitters in the field are more likely to be old.

Despite this decline in activity there are many reports of flare activity even among L dwarfs. For very late-type $\mathrm{M}$ dwarfs Gizis et al. (2000) estimated a flaring time fraction of about $7 \%$, while Liebert et al. (2003) found a flare duty cycle of about $1 \%$ for L dwarfs, suggesting that there must be some ongoing magnetic activity in these dwarfs despite the absence of a persistent chromosphere or corona.

In this paper we report on a huge flare on DENIS 1048-39 detected in the $\mathrm{H}_{\alpha}, \mathrm{H}_{\beta}$, and sodium emission lines. In Sect. 2 we describe the VLT data and their analysis, in Sect. 3 we discuss the timing behaviour of the flare.

\section{Observations and data analysis}

The observations reported in this paper were carried out with ESO's Kueyen telescope at Paranal equipped with the Ultraviolet-Visual Echelle Spectrograph (UVES) on March, 14th, 2002. Four spectra of DENIS 1048-39 were taken in sequence, each with an exposure time of $1200 \mathrm{~s}$. The individual observations started at 04:12:46, 04:33:43, 04:54:40 and 
05:22:40 UT. The instrument was operated in dichroic mode, yielding 33 echelle orders in the blue arm (spectral coverage from 3030 to $3880 \AA$ ) and 39 orders in the red arm (spectral coverage from 4580 to $6680 \AA$ ) with a typical resolution of $\sim 45000$. The red part of the spectrum was recorded on two separate CCDs; therefore there is a spectral gap from $\sim 5640$ to $5740 \AA$ resulting from the spatial separation of the CCDs. As a consequence of the dichroic mode used we did not cover the lines from $\mathrm{H}_{3}$ up to $\mathrm{H}_{8}$ of the Balmer series, nor the $\mathrm{Ca}$ II $\mathrm{H}$ and $\mathrm{K}$ lines.

The data were reduced using IRAF. The wavelength calibration was carried out with thorium-argon spectra with an accuracy of $\sim 0.03 \AA$ in the blue arm and $\sim 0.05 \AA$ in the red arm.

The fitting of the spectral lines was carried out with the CORA fit program (Ness \& Wichmann 2002), kindly provided to us by Dr. Jan-Uwe Ness and originally developed for analyzing high resolution X-ray spectra. The fit algorithms employed by CORA are also well suited for a modeling of all types of emission lines with count statistics. CORA uses a maximum likelihood method for the fitting of line profiles. It contains a number of tools for modeling blended lines, allowing the user to specify the number of lines to be modeled, whether the line shapes should be individually fitted or fitted with a fixed line profile, and whether the line centroids are allowed to vary freely or are fixed with respect to each other. The program also provides an accurate error analysis.

\section{The UVES spectrum of DENIS 1048-39}

\subsection{Emission line variability}

In our UVES spectra of DENIS 1048-39 we find three prominent emission lines in the red part of the spectra, which can be clearly identified as $\mathrm{H}_{\alpha}, \mathrm{H}_{\beta}$, and the $\mathrm{Na}$ I D doublet at 5889 and $5895 \AA$. In Figs. 1-3 we plot the spectral region around $\mathrm{H}_{\alpha}$, $\mathrm{H}_{\beta}$, and the $\mathrm{Na} \mathrm{I} \mathrm{D}$ for the four individual spectra. In order to facilitate the comparison between the spectra we also plot the averaged spectrum as a dotted line. Note that the spectra are not (yet) shifted to the star's rest frame.

In the spectral sequence of our VLT observations a clear intensity increase as well as a blue shift of the $\mathrm{H}_{\alpha}$ and $\mathrm{H}_{\beta}$ lines is seen. In the last spectrum of our series an extremely broad emission feature appears, centered at about $6560.5 \AA$ on the blue side of the $\mathrm{H}_{\alpha}$ line. A similar emission feature appears for the $\mathrm{H}_{\beta}$ line centered at about $4860 \AA$. If interpreted as Doppler shift, this would imply emission from material moving with a velocity of $100 \mathrm{~km} \mathrm{~s}^{-1}$ towards the observer.

For the Na I D lines the flux is not constant, but no clear trend can be seen. The stellar Na I D lines are heavily blended with terrestrial airglow lines of $\mathrm{NaI} \mathrm{D}$; in addition, there are further airglow lines in this spectral region at $5888.19 \AA$ and 5894.47 $\AA$, well known to occur in the UVES instrument (Hanuschik 2003). The blue shift of the stellar Na I D lines can be unambiguously seen, when the stellar Na I D line peak moves out of the stationary airglow lines in the last spectrum. We therefore conclude that the recorded line shifts cannot be due to incorrect wavelength calibrations.

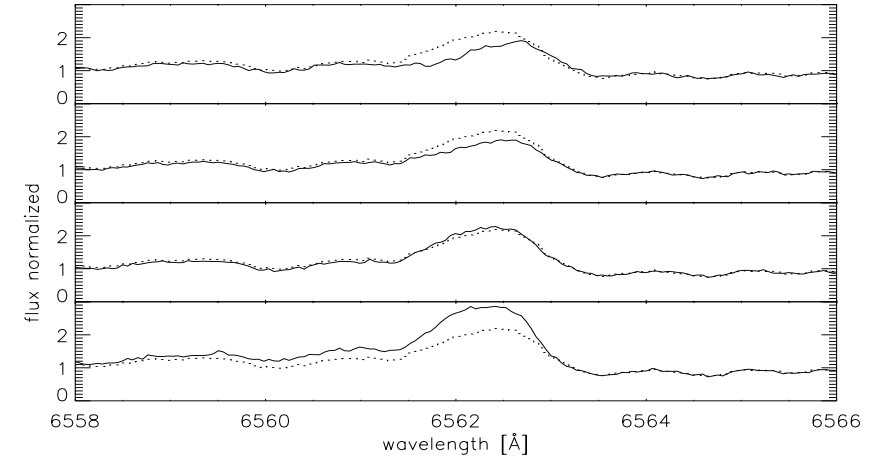

Fig. 1. Time series of the $\mathrm{H}_{\alpha}$-line first spectrum at the top, last spectrum at the bottom. The dotted spectrum is the time averaged spectrum of DENIS 1048-39. Notice the gradual increase and blue shift of the line; in the last spectrum a broad feature is seen to emerge on the blue side of the line centered on about $6560.5 \AA$.

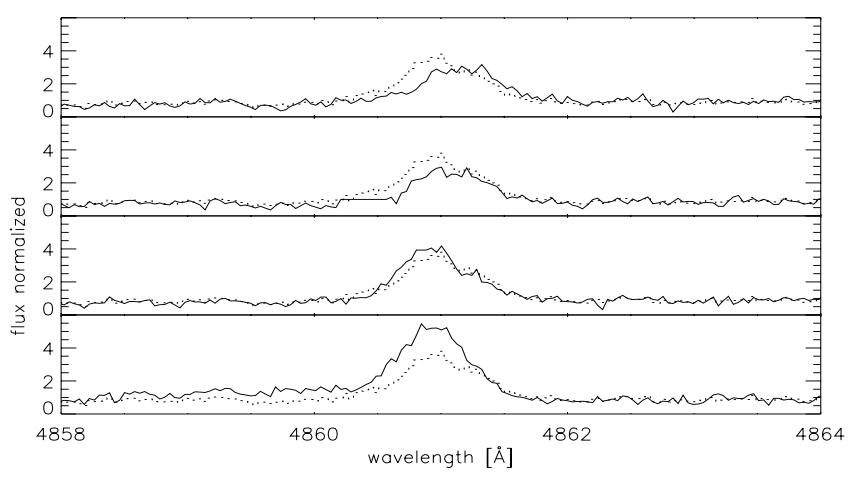

Fig. 2. Time series of the $\mathrm{H}_{\beta}$-line as in Fig. 1. Again a blue shift of the line can be seen as well as a broad emission feature at the blue side of the line in the last spectrum. In the spectra some cosmics were replaced manually by a horizontal line. The dotted line is again the time averaged spectrum.

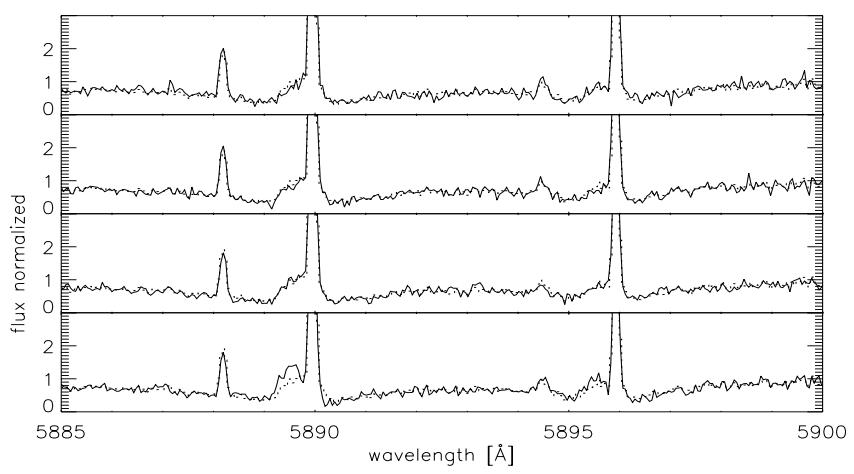

Fig. 3. Time series of the Na I D lines as in Fig. 1. The dotted line denotes again the time averaged spectrum. Note the blue shift that can be seen in comparison to the airglow Na I D lines especially in the last spectrum, where the peak of the stars Na I D lines moves out of the airglow lines.

In Fig. 4 we plot the time series of the spectrum between $5390 \AA-5420 \AA$, which is purely of photospheric origin. This spectral region obviously remains constant and thus the photospheric continuum appears to be unaffected by the flaring emission lines. 
Table 1. Properties of the Na I D lines determined from fitting the Na I D airglow line and the star's Na I D line simultaneously. Quoted are all free fit parameters and the line shift of the star's Na I D line. Due to the heavy blending the error estimation of the half width could not be done for each spectrum.

\begin{tabular}{|c|c|c|c|c|c|}
\hline & & Star & Airglow & Star & Airglow \\
\hline \multirow[t]{4}{*}{ 1st spectrum } & $\lambda_{\text {cen }}[\AA]$ & $5890.00 \pm 0.04$ & $5890.19 \pm 0.02$ & $5895.96 \pm 0.03$ & $5896.17 \pm 0.02$ \\
\hline & $\sigma[\AA]$ & $0.30 \pm 0.03$ & $0.05 \pm 0.01$ & 0.25 & $0.05 \pm 0.01$ \\
\hline & Amplitude [electrons] & $1920 \pm 73$ & $5890 \pm 85$ & $1225 \pm 65$ & $3665 \pm 69$ \\
\hline & Line shift $\left[\mathrm{km} \mathrm{s}^{-1}\right]$ & $2.5 \pm 2.0$ & & $1.8 \pm 1.1$ & \\
\hline \multirow[t]{4}{*}{ 2nd spectrum } & $\lambda_{\text {cen }}[\AA]$ & $5889.97 \pm 0.03$ & $5890.20 \pm 0.03$ & $5895.91 \pm 0.04$ & $5896.16 \pm 0.02$ \\
\hline & $\sigma[\AA]$ & $0.28 \pm 0.01$ & $0.06 \pm 0.01$ & $0.24 \pm 0.02$ & $0.06 \pm 0.01$ \\
\hline & Amplitude [electrons] & $2110 \pm 74$ & $5258 \pm 82$ & $942 \pm 60$ & $3359 \pm 66$ \\
\hline & Line shift $\left[\mathrm{km} \mathrm{s}^{-1}\right]$ & $1.0 \pm 1.5$ & & $-0.71 \pm 2.0$ & \\
\hline \multirow[t]{4}{*}{ 3rd spectrum } & $\lambda_{\text {cen }}[\AA]$ & $5889.92 \pm 0.02$ & $5890.20 \pm 0.01$ & $5895.86 \pm 0.05$ & $5896.17 \pm 0.01$ \\
\hline & $\sigma[\AA]$ & $0.24 \pm 0.01$ & $0.06 \pm 0.01$ & 0.19 & $0.06 \pm 0.01$ \\
\hline & Amplitude [electrons] & $2316 \pm 73$ & $5208 \pm 81$ & $811 \pm 54$ & $3475 \pm 66$ \\
\hline & Line shift $\left[\mathrm{km} \mathrm{s}^{-1}\right]$ & $-1.5 \pm 1.0$ & & $-3.3 \pm 2.5$ & \\
\hline \multirow[t]{4}{*}{ 4th spectrum } & $\lambda_{\text {cen }}[\AA]$ & $5889.76 \pm 0.04$ & $5890.19 \pm 0.02$ & $5895.78 \pm 0.07$ & $5896.17 \pm 0.02$ \\
\hline & $\sigma[\AA]$ & $0.19 \pm 0.01$ & $0.06 \pm 0.01$ & $0.14 \pm 0.01$ & $0.05 \pm 0.01$ \\
\hline & Amplitude [electrons] & $2273 \pm 62$ & $5069 \pm 76$ & $832 \pm 47$ & $2977 \pm 60$ \\
\hline & Line shift $\left[\mathrm{km} \mathrm{s}^{-1}\right]$ & $-9.7 \pm 2.0$ & & $-7.3 \pm 3.6$ & \\
\hline
\end{tabular}

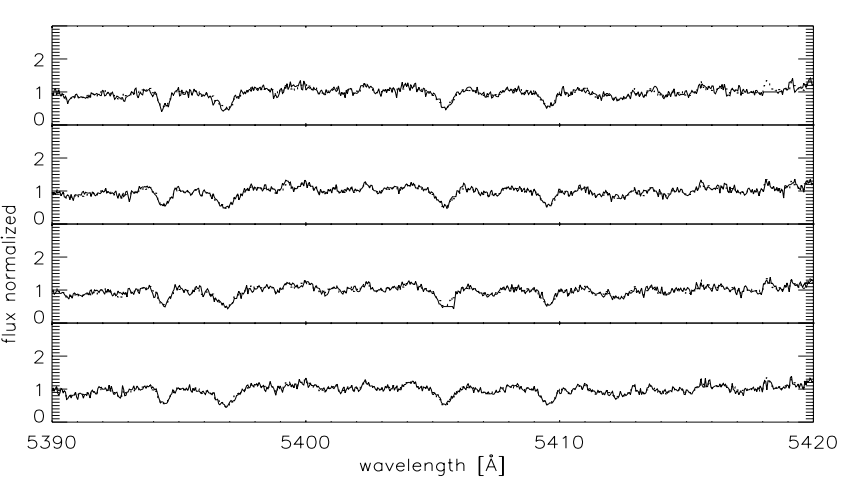

Fig. 4. Timeseries of a spectral range with several metal absorption lines. Clearly these photospheric lines are not affected by the blue shift seen in the chromospheric emission lines. Two cosmics were removed manually by a horizontal line. The four pronounced absorption lines are due to Mn I (5394 ̊), Fe I (5397 and $5405 \AA)$ and Cr I (5409 $\AA$ ). Again the dotted line denotes the time averaged spectrum.

\subsection{Radial and rotational velocity}

Having confirmed that the absorption lines are not affected by any blue shifts, we measured the radial velocity of the star to be $-9.6 \pm 2.0 \mathrm{~km} \mathrm{~s}^{-1}$ in agreement with the value of $-10.1 \pm 0.5 \mathrm{~km} \mathrm{~s}^{-1}$ found by Delfosse et al. (2001). All line fitting with CORA was done after shifting the spectra to the rest frame. In addition to the radial velocity we also measured the rotational velocity of DENIS 1048-39 using 7 apertures in the red arm of the spectrum without any emission and airglow lines. We used the spectrum of the M9 star LHS 2065 (taken during the same observation run) as a template and convolved it with rotational velocities from 3 up to $40 \mathrm{~km} \mathrm{~s}^{-1}$. The best rotational fit of the spun-up template and DENIS 1048-39 was determined with a $\chi^{2}$ test for every aperture used. The values determined for the different apertures were then averaged to yield a final (averaged) rotational velocity of $25.0 \pm 2 \mathrm{~km} \mathrm{~s}^{-1}$ in good agreement with the $v \sin (i)$-value of $27 \pm 5 \mathrm{~km} \mathrm{~s}^{-1}$ found by Delfosse et al. (2001).

\subsection{The NaI $D$ lines}

We fitted the stellar and airglow lines of Na I D simultaneously treating the central wavelength $\lambda_{\text {cen }}$, Gaussian $\sigma$ and amplitude for every line as free parameters. The fit results are listed in Table 1. While the central wavelength of the airglow lines is stable as it should, our fits indicate a steady bluewards drift in the stellar Na I D lines. The line shifts were computed using 5889.950 and $5895.924 \AA$ as reference central wavelengths; although the errors are large, the trend to bluer line centers is clearly visible.

The large amplitude for the line at 5895.924 $\AA$ in the first spectrum does not agree with the line amplitudes of the other three spectra that vary only slightly. We ascribe this to the heavy blending of the line and the faintness of the feature which makes its amplitude sensitive to noise and to the background level chosen for the fit.

\subsection{The $H_{\alpha}$ and the $H_{\beta}$ line}

The amplitudes of the $\mathrm{H}_{\alpha}$ and $\mathrm{H}_{\beta}$ lines were fitted with a single Gaussian line profile as for the Na I D lines. For the $\mathrm{H}_{\beta}$ line this seems to be a reasonably good description although the line center shows some fine structure. However, for the $\mathrm{H}_{\alpha}$ line these fits yielded only a very poor description of line profiles in UVES data. A major problem for our fits of the $\mathrm{H}_{\alpha}$ line is the background treatment, which is somewhat complicated with several unidentified broad emission and absorption features on 
Table 2. Properties of the $\mathrm{H}_{\alpha}$ and the $\mathrm{H}_{\beta}$ line. The line shift is given in $\mathrm{km} \mathrm{s}^{-1}$ using 4861.332 and $6562.817 \AA$ as reference central wavelength.

\begin{tabular}{|c|c|c|c|c|c|c|}
\hline & & \multicolumn{4}{|c|}{$\mathrm{H}_{\alpha}$} & \multirow[t]{2}{*}{$\mathrm{H}_{\beta}$} \\
\hline & & Bump & 1 st comp & 2nd comp & Total & \\
\hline \multirow[t]{4}{*}{ 1st spectrum } & $\lambda_{\text {cen }}[\AA]$ & $6561.29 \pm 0.04$ & $6562.62 \pm 0.01$ & $6563.07 \pm 0.01$ & & $4861.36 \pm 0.12$ \\
\hline & $\sigma[\AA]$ & $0.54 \pm 0.04$ & $0.34 \pm 0.01$ & $0.22 \pm 0.01$ & & $0.31 \pm 0.02$ \\
\hline & Amplitude [electrons] & $7477.3 \pm 237.2$ & $14094.2 \pm 259.0$ & $7357.9 \pm 211.0$ & $21452 \pm 334$ & $4032 \pm 81$ \\
\hline & Line shift $\left[\mathrm{km} \mathrm{s}^{-1}\right]$ & & $-9.0 \pm 0.5$ & $11.6 \pm 0.5$ & & $1.7 \pm 7.4$ \\
\hline \multirow[t]{4}{*}{ 2nd spectrum } & $\lambda_{\text {cen }}[\AA]$ & $6561.19 \pm 0.05$ & $6562.40 \pm 0.01$ & $6562.96 \pm 0.01$ & & $4861.29 \pm 0.10$ \\
\hline & $\sigma[\AA]$ & $0.40 \pm 0.01$ & $0.42 \pm 0.01$ & $0.25 \pm 0.01$ & & $0.26 \pm 0.02$ \\
\hline & Amplitude [electrons] & $8003.8 \pm 219.3$ & $22086.6 \pm 301.1$ & $10642.6 \pm 232.5$ & $32728 \pm 380$ & $3730 \pm 78$ \\
\hline & Line shift $\left[\mathrm{km} \mathrm{s}^{-1}\right]$ & & $-19.0 \pm 0.5$ & $6.5 \pm 0.5$ & & $-2.6 \pm 6.1$ \\
\hline \multirow[t]{4}{*}{ 3rd spectrum } & $\lambda_{\text {cen }}[\AA]$ & $6561.13 \pm 0.05$ & $6562.36 \pm 0.01$ & $6562.92 \pm 0.01$ & & $4861.15 \pm 0.10$ \\
\hline & $\sigma[\AA]$ & $0.36 \pm 0.01$ & $0.40 \pm 0.01$ & $0.26 \pm 0.01$ & & $0.28 \pm 0.01$ \\
\hline & Amplitude [electrons] & $7669.0 \pm 213.6$ & $33246.9 \pm 325.3$ & $15394.6 \pm 261.5$ & $48642 \pm 416$ & $6460.9 \pm 97.2$ \\
\hline & Line shift $\left[\mathrm{km} \mathrm{s}^{-1}\right]$ & & $-21.3 \pm 0.5$ & $4.7 \pm 0.5$ & & $-11.2 \pm 6.1$ \\
\hline \multirow[t]{4}{*}{ 4th spectrum } & $\lambda_{\text {cen }}[\AA]$ & $6561.19 \pm 0.01$ & $6562.39 \pm 0.01$ & $6562.93 \pm 0.01$ & & $4861.14 \pm 0.02$ \\
\hline & $\sigma[\AA]$ & $0.54 \pm 0.01$ & $0.36 \pm 0.01$ & $0.22 \pm 0.01$ & & $0.26 \pm 0.01$ \\
\hline & Amplitude [electrons] & $22450 \pm 288.5$ & $43569.8 \pm 340.2$ & $16920 \pm 253.5$ & $60490 \pm 423$ & $7841.0 \pm 107.1$ \\
\hline & Line shift $\left[\mathrm{km} \mathrm{s}^{-1}\right]$ & & $-19.4 \pm 0.5$ & $4.7 \pm 0.5$ & & $-11.8 \pm 1.2$ \\
\hline
\end{tabular}

the blue side of the line and a slightly higher background level on the blue compared to the red side. Although there appears to be some variation in the background level we decided to use a constant background since there is no other reasonable method to treat the background problem i.e., there is no obvious gradient. We used the background value redwards of the line found in the second spectrum for our fits. Therefore the first broad emission feature at $6561.29 \AA$ (see Fig. 5) must be treated as an additional line feature in the fit of the $\mathrm{H}_{\alpha}$ line. The $\mathrm{H}_{\alpha}$ line also shows a strong asymmetry in the blue wing, where more and more flux appears during our spectral sequence, while the red wing of the line stays almost constant. We therefore decided to use two spectral components to describe the shape of the line core and a third component to account for the closest blue emission feature in the background. All fit components were assumed to be Gaussians and we use again central wavelength $\lambda_{\text {cen }}$, Gaussian $\sigma$ and the line amplitude as free fit parameters. The results of our fits of the $\mathrm{H}_{\alpha}$ and the $\mathrm{H}_{\beta}$ line are listed in Table 2 . In order to provide an example of the quality of our fits we show the fitted $\mathrm{H}_{\alpha}$ line for the first spectrum in Fig. 5. While not necessarily physical, the three components do obviously provide a good analytical description of the recorded UVES spectrum.

Since two Gaussian components are required for the fit of the core of the $\mathrm{H}_{\alpha}$ line, the question arises, why the $\mathrm{H}_{\beta}$ line behaves differently and can be fitted with a single component. Indeed, a close inspection of the line shape of the $\mathrm{H}_{\beta}$ line in the third spectrum also shows an additional red component. Therefore we tried to fit the $\mathrm{H}_{\beta}$ line with two components as well. For the third spectrum this leads to a substantial improvement of the fit resulting in similar line shifts and a similar ratio of the two components as for $\mathrm{H}_{\alpha}$ (see Fig. 6); unfortunately for the other spectra a fit with two components describing the line core does not lead to unique solutions and does not provide

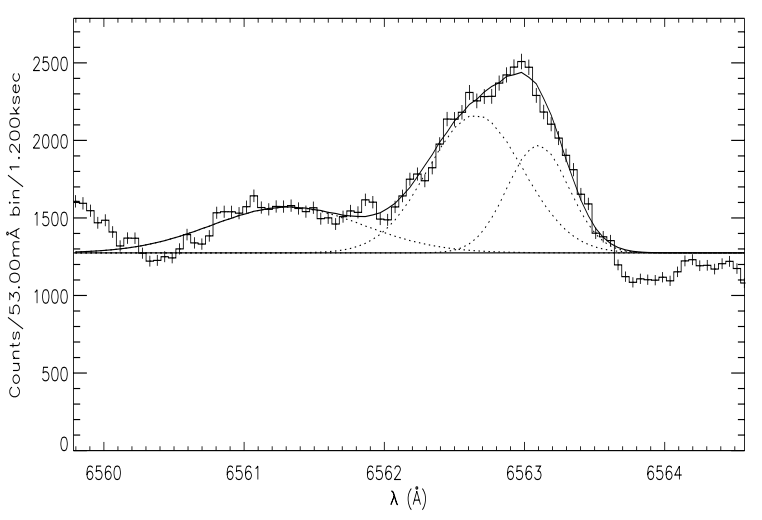

Fig. 5. Fit of the $\mathrm{H}_{\alpha}$ line in the first spectrum of the series. Two fit components are used for the line, while the third component accounts for the bump in the continuum blue to the line. The horizontal line is the background used for the fit. The dotted lines mark the single gaussian components used for the fit. Their free fit parameters can be found in Table 2.

significantly improved fit results. Therefore we decided to use the fits with one component for $\mathrm{H}_{\beta}$ bearing in mind that there is evidence for a second red component of the line that behaves similarly to the red component of the $\mathrm{H}_{\alpha}$ line at least for the third spectrum.

Our fit results for the $\mathrm{H}_{\alpha}$ line clearly demonstrate that the reddest line component is only weakly blue shifted during our observations, while the blue component is shifted by $12.3 \mathrm{~km} \mathrm{~s}^{-1}$ bluewards from its initial position during the first three spectra. In the last spectrum there are no additional line shifts observed neither for the $\mathrm{H}_{\alpha}$ line components nor for the $\mathrm{H}_{\beta}$ line, rather a substantial flux increase occurs in the blue component of the $\mathrm{H}_{\alpha}$ line and in the $\mathrm{H}_{\beta}$ line. For the $\mathrm{H}_{\alpha}$ line the ratio between the amplitude of the two components is approximately constant for the first three spectra, but in the last 


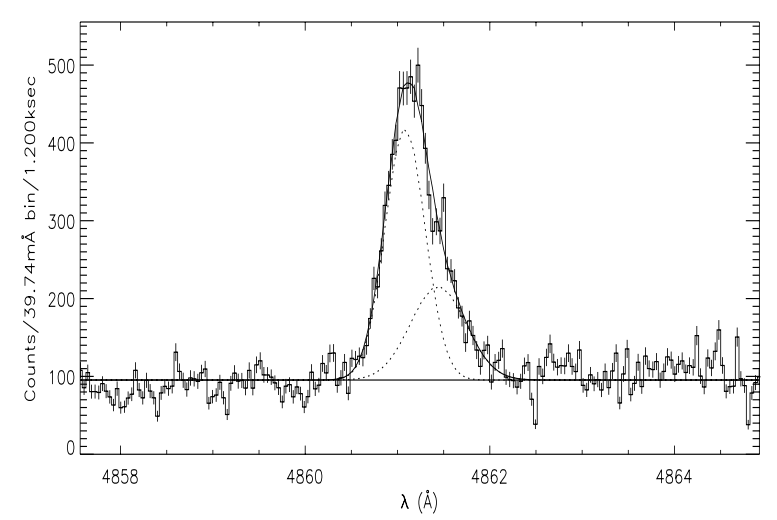

Fig. 6. Fit of the $\mathrm{H}_{\beta}$ line in the third spectrum of the series. Two fit components are used for the line resulting in a good fit of the line especially in the red wing. The horizontal line is the background used for the fit. The dotted lines mark the single Gaussian components.

Table 3. Properties of the broad emission feature bluewards of the $\mathrm{H}_{\alpha}$ and the $\mathrm{H}_{\beta}$ line in the last spectrum. The line shift is given in $\mathrm{km} \mathrm{s}^{-1}$ using 4861.332 and $6562.817 \AA$ as reference central wavelength.

\begin{tabular}{ccc}
\hline \hline & $\mathrm{H}_{\alpha}$ & $\mathrm{H}_{\beta}$ \\
\hline$\lambda_{\text {cen }}[\AA]$ & $6560.51 \pm 0.01$ & $4859.76 \pm 0.11$ \\
$\sigma[\AA]$ & $1.30 \pm 0.01$ & $0.80 \pm 0.03$ \\
Amplitude [electrons] & $27347.1 \pm 437.1$ & $3645.3 \pm 104.7$ \\
Line shift [km s$\left.{ }^{-1}\right]$ & $-109.9 \pm 0.5$ & $-96.9 \pm 6.8$ \\
\hline
\end{tabular}

spectrum the blue component is observed with some excess flux. We also note that the line shifts measured for the $\mathrm{H}_{\beta}$ line are consistent with the line shifts found in the $\mathrm{Na}$ I D lines.

In the last spectrum of our spectral series a tremendous flux increase occurs bluewards of the $\mathrm{H}_{\alpha}$ line. This increase can be seen in Fig. 1, but most clearly in Fig. 7. The "background" on the blue and red side of the $\mathrm{H}_{\alpha}$ line agrees for all spectra except for the blue side of the last spectrum in the spectral series. There a flux is detectable out to $5 \AA$ from line center, while blueward of $6558 \AA$ the two background levels are identical again.

In order to fit this additional flux in the last spectrum we used the first spectrum of the series as a background "template". Obviously with this strategy we will not obtain meaningful results for the fit of the $\mathrm{H}_{\alpha}$ line itself, however, we merely intend to characterize the flux increase on the blue side of the $\mathrm{H}_{\alpha}$ line. For the fit we used two Gaussian components with the same free fit parameters as before. Since the fit of the $\mathrm{H}_{\alpha}$ line core is ill defined due to the background anyway we only used one Gaussian component for this core, while the other Gaussian is used to fit the broad emission component (see Fig. 7).

For the $\mathrm{H}_{\beta}$ in the last spectrum there is a similar broad emission feature of the blue side of the line as can be seen from Fig. 2. In this case the surrounding background is quite flat, therefore a fit of this broad emission line with a constant background would have been possible but for consistency reasons we fitted the broad emission feature with the first spectrum as background, too. The best fit parameters can be found in Table 3.

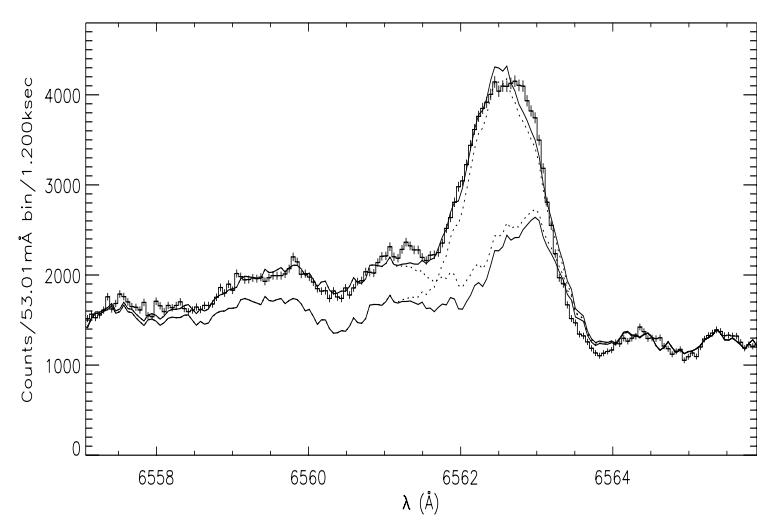

Fig. 7. Fit of the broad emission feature blueward of the $\mathrm{H}_{\alpha}$ line in the last spectrum of the series. The lower black line is the first spectrum of the series that was used as background. Note the flux increase between the two spectra not only in the line core but also in the broad emission feature centered at $6560.5 \AA$ blueward of the line. The two dotted lines are the single gaussian components used for the fit. Since the background is variable their shape does not appear to be Gaussian. Their free fit parameters can be found in Table 3 .

The line shifts of the broad emission features bluewards of the $\mathrm{H}_{\alpha}$ and the $\mathrm{H}_{\beta}$ line are not in agreement with each other due to the small error for the $\mathrm{H}_{\alpha}$ line $\left(0.5 \mathrm{~km} \mathrm{~s}^{-1}\right)$. In contrast to this the error for the line shift of the broad component corresponding to the $\mathrm{H}_{\beta}$ line is quite big $\left(6.8 \mathrm{~km} \mathrm{~s}^{-1}\right)$. Both errors should be considered only as formal ones since they are only statistical errors. The unusual background treatment can lead to additional systematic errors. If the errors for the $\mathrm{H}_{\alpha}$ line are as large as that of the $\mathrm{H}_{\beta}$ line, the line shifts actually agree very well.

Since absolute flux measurements were not the primary goal of this observing run, we obtained only a few measurements of standard stars. We calibrated our spectra with the standard star HD 49798, estimating the errors in the resulting fluxes to be as large as a factor of two. The measured line counts of the two main components of the $\mathrm{H}_{\alpha}$ line then translate into a flux of $6.3 \times 10^{-14} \mathrm{erg} \mathrm{cm}^{-2} \mathrm{~s}^{-1}$, resulting in a line luminosity of $1.6 \times 10^{26} \mathrm{erg} \mathrm{s}^{-1}$ using a distance of $4.6 \mathrm{pc}$. This is not particularly large compared to other $\mathrm{H}_{\alpha}$ line luminosities found for example for 2MASSW J0149090+295613 to be $15.3 \times 10^{26} \mathrm{erg} \mathrm{s}^{-1}$ during a flare (Liebert et al. 1999). Estimating the bolometric luminosity with $M_{J}=11.28 \mathrm{mag}$ (Neuhäuser et al. 2002) and $\mathrm{BC}=1.95$ mag leads to $L_{\mathrm{bol}}=$ $1.5 \times 10^{30} \mathrm{erg} \mathrm{s}^{-1}$ which yields a ratio of $\log \left(L_{\mathrm{H} \alpha} / L_{\mathrm{bol}}\right)=-4.0$. According to Liebert et al. (2003) this would be quite high for an M9 dwarf in quiescent emission, but it is well in the range of observed $L_{\mathrm{H} \alpha}$ to $L_{\mathrm{bol}}$ ratios for the few flaring events known for objects at the M to L spectral class transition.

\subsection{Other Balmer lines}

Due to the chosen instrumental setup we do not cover the $\mathrm{H}_{3}$ up to $\mathrm{H}_{8}$ of the Balmer series. Therefore we searched for emission lines at the position of $\mathrm{H}_{9}$ and higher Balmer series members. Unfortunately the blue part of the spectra is very noisy since the count rate is very low. We do find a faint emission 


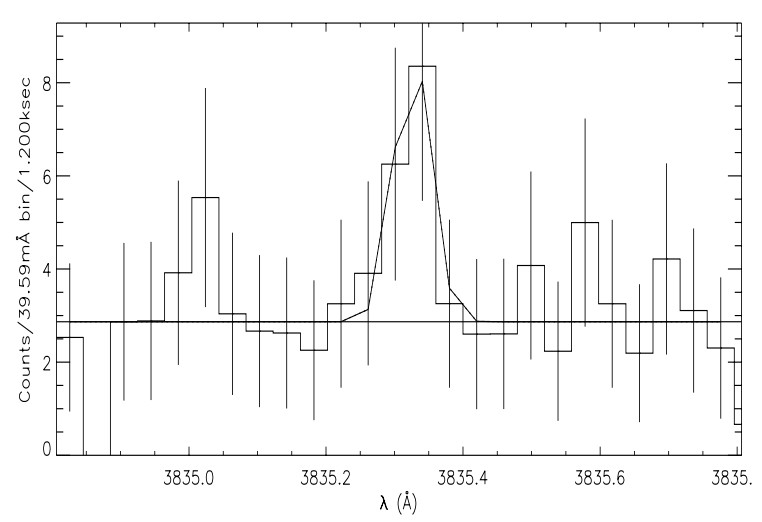

Fig. 8. The last spectrum in the series around $3835.386 \AA$ A. The fit shows the faint emission line of $\mathrm{H}_{9}$. The horizontal line is the background used for the fit.

line at $3835.31 \pm 0.04 \AA$ in our last spectrum, corresponding to a blue shift of $-5.9 \pm 3.1 \mathrm{~km} \mathrm{~s}^{-1}$ which is less than that of the $\mathrm{H}_{\beta}$ line. Since $\mathrm{H}_{\alpha}$ and $\mathrm{H}_{\beta}$ differ significantly in appearance and blueshift, an interpretation of this faint line as $\mathrm{H}_{9}$ is very attractive. We found the best fit with an amplitude of $9.1 \pm$ 4.0 electrons, $\sigma$ of $0.02 \pm 0.01 \AA$ and central wavelength of $3835.31 \pm 0.04 \AA$. The line fit can be seen in Fig. 8. We also searched for higher Balmer lines. In the last spectrum an emission feature at $3797.76 \pm 0.12 \AA$ corresponding to a blue shift of $-11.0 \pm 9.5 \mathrm{~km} \mathrm{~s}^{-1}$ of $\mathrm{H}_{10}$ is found, but it is so weak that it can be due to noise. On the wavelength positions of $\mathrm{H}_{11}$ no emission is found.

\section{Discussion and conclusions}

\subsection{Emission geometry}

The measured half widths of the $\mathrm{Na}$ I $\mathrm{D}$ and the $\mathrm{H}_{\beta}$ lines always stay below $20 \mathrm{~km} \mathrm{~s}^{-1}$, i.e., they are smaller than the measured rotational velocity. The same applies to the two main components of the $\mathrm{H}_{\alpha}$ line. This suggests that the emission for the Balmer lines as well as for the $\mathrm{NaI} \mathrm{D}$ lines is confined to a restricted region on the star. Since the $\mathrm{H}_{\alpha}$ line consists of two components and there is evidence that the $\mathrm{H}_{\beta}$ line consists of two components as well, the star could host even two active regions producing the observed Balmer emission. Now the question arises what the nature of these two active regions may be. There are two main possibilities: a dynamic scenario or a static scenario.

\subsection{Static or dynamic scenario?}

The dynamic scenario invokes two active regions located on the surface of the star and mass motions within these active regions. The Balmer emission comes from the chromosphere, nevertheless very close to the surface of the star. At least in one of the two regions a brightening in the Balmer lines takes place on a timescale of about $1.5 \mathrm{~h} . \mathrm{H}_{\alpha}$ and $\mathrm{H}_{\beta}$ emitting material moves towards the observer and causes the blue shift.

The static scenario interprets the two regions as emitting gas clouds corotating in some distance to the stars surface as was first proposed for the K0 star AB Dor by Collier Cameron \& Robinson (1989). Since for one component the line shift becomes bluer this one must be rising while the other one must be about to set behind the star since its line shift is moving towards the blue. The blue shifted component must then be captured during its rise above the horizon of the star to account for the flux increase. This latter static scenario can be excluded because of the rapid rotation of the stars as follows: for an estimated radius of $R_{\star}=0.1 R_{\odot}$ the maximal rotation period of DENIS 1048-39 is $4.9 \mathrm{~h}$. Therefore in the four consecutive spectra lasting together $80 \mathrm{~min}$ the star completes about a fourth of its rotation. If the emitting gas is confined in a corotating cloud, the measured velocity shift should exceed the star's rotational velocity in at least one spectrum for orbits near the equatorial plane. Moreover the flux increase cannot be explained by a cloud rotating into view because the star rotates too fast for this interpretation. A more natural explanation is therefore the scenario of active regions with mass motions at the surface of the star that brighten during the spectral series.

\subsection{Blue-shifted Balmer emission}

Let us now consider the broad emission features bluewards of the two Balmer lines seen only in the fourth spectrum. Again a dynamic or static scenario may be considered. Let us first assume a static interpretation with a corotating cloud. Since its radial velocity of about $100 \mathrm{~km} \mathrm{~s}^{-1}$ is too high to be interpreted by a region on or near the surface of the star, the emission would have to come from a cloud at some distance to the star. Such a cloud must be confined then by magnetic loops above the surface of the star and has rotated just into the field of view. If one assumes a stellar inclination close to $90^{\circ}$ and a cloud in the equatorial plane, one can compute its distance $R$ from the rotation axis with $R=\frac{v P}{2 \pi}$ with $v$ denoting the radial velocity and $P$ the rotation period of DENIS 1048-39. We find a distance of $R=4 R_{\star}$ with the measured radial velocity of $100 \mathrm{~km} \mathrm{~s}^{-1}$, which is below the Keplerian corotation radius of $6 R_{\star}$ like the clouds found on $\mathrm{AB}$ Dor in $\mathrm{H}_{\alpha}$ (Donati \& Collier Cameron 1997) and other chromospheric lines (Brandt et al. 2001). But since the star rotates fast, the feature should be visible in more than one spectrum since for its distance of four stellar radii it can be shadowed by the star only about $10 \%$ of the time, i.e. about $30 \mathrm{~min}$, thus again a dynamic interpretation appears more likely.

In the dynamical interpretation the line shift is explained in terms of material ejected by the star. Since the emission in the main body of the $\mathrm{H}_{\alpha}$ brightens substantially in the same spectrum it is suggestive that the broad emission feature is connected to the active region producing the $\mathrm{H}_{\alpha}$ line arguing further in favor of a dynamic scenario. In the context of interpreting the broad emission feature as the signature of a rising cloud the question remains unsettled whether this rise leads to mass ejection since we do not know the longitude of the cloud. Assuming again an radius of $0.1 R_{\odot}$ we find an escape velocity of about $550 \mathrm{~km} \mathrm{~s}^{-1}$ for a mass between 0.075 and $0.09 M_{\odot}$ while the projected velocity of the cloud is about $100 \mathrm{~km} \mathrm{~s}^{-1}$. 
Let us now consider the width of the emission feature. This width can be due to temperature and turbulent broadening if one thinks of a confined gas cloud rising towards the observer. A first estimate of the temperature of a $\mathrm{H}_{\alpha}$ emitting gas is about $10000 \mathrm{~K}$ leading to a thermal broadening of $9 \mathrm{~km} \mathrm{~s}^{-1}$, far less than the observed total line broadening of $50 \mathrm{~km} \mathrm{~s}^{-1}$. In this scenario with a rising cloud one can estimate the height of the cloud after the twenty minutes exposure. Assuming a constant cloud velocity of the cloud and an ejection start right at the beginning of the exposure leads to a cloud height of $1.7 R_{\star}$. It is probable that the cloud has been decelerated during such a rise. Therefore a second interpretation of the line width is that different velocities of the cloud during deceleration are integrated over the exposure time. Moreover, the cloud may consist of more than one component with different velocities.

Besides these uncertainties in the event geometry there is no doubt that a dynamical interpretation of the spectra is needed. The last spectrum is then quite suggestively interpreted as the onset of a flare on DENIS 1048-39 as reported for AD Leo by Houdebine et al. (1990) who found a similar flux enhancement in the far blue wing of the $\mathrm{H}_{\gamma}$ line during a particular violent flare on AD Leo. Since these authors found projected velocities of up to $5800 \mathrm{~km} \mathrm{~s}^{-1}$ this event was clearly associated with a mass ejection, while this question remains unsettled for DENIS 1048-39.

\subsection{Summary}

In conclusion, we find at least two active regions on DENIS 1048-39 contributing to the bulk of the Balmer line flux. Mass motions directed towards the observer are found for the emission lines of the Balmer series as well as for the $\mathrm{Na} \mathrm{D}$ lines. In the last spectrum of our observations the onset of a flare seems to take place, since substantial brightening and blueshifts can be seen in the lines. In addition in the $\mathrm{H}_{\alpha}$ and the $\mathrm{H}_{\beta}$ line there is a broad emission feature on the blue side of the line. This can be interpreted as a rising cloud.

Since DENIS 1048-39 seems to be located directly at the hydrogen burning limit this flare gives evidence that such events may be more ubiquitous than previously assumed. It is consistent with X-ray detections of brown dwarfs (Mokler \& Stelzer 2002) and the X-ray flare event found on the similar late-type star LHS 2065 (Schmitt \& Liefke 2002).

\section{References}

Brandt, J. C., Heap, S. R., Walter, F. M., et al. 2001, AJ, 121, 2173

Collier Cameron, A., \& Robinson, R. D. 1989, MNRAS, 236, 57

Delfosse, X., Forveille, T., Martín, E. L., et al. 2001, A\&A, 366, L13

Donati, J.-F., \& Collier Cameron, A. 1997, MNRAS, 291, 1

Epchtein, N. 1997, in The Impact of Large Scale Near-IR Sky Surveys, ed. F. Garzonet al (Kluwer: Dordrecht), ASSL, 210, 15

Gizis, J. E., Monet, D. G., Reid, I. N., et al. 2000, AJ, 120, 1085

Hanuschik, R. W. 2003, A\&A, 407, 1157

Houdebine, E. R., Foing, B. H., \& Rodono, M. 1990, A\&A, 238, 249

Liebert, J., Kirkpatrick, J. D., Cruz, K. L., et al. 2003, AJ, 125, 343

Liebert, J., Kirkpatrick, J. D., Reid, I. N., \& Fisher, M. D. 1999, ApJ, 519,345

Mokler, F., \& Stelzer, B. 2002, A\&A, 391, 1025

Ness, J.-U., \& Wichmann, R. 2002, AN, 323, 129

Neuhäuser, R., Guenther, E. W., Alves, J., et al. 2002, AN, 323, 447

Schmitt, J. H. M. M., \& Liefke, C. 2002, A\&A, 382, L9 